\title{
不同结构铝氧化物对磷的吸附特征
}

\author{
李 敬 ${ }^{1,2}$, 刘晓月 ${ }^{2}$, 邱千峰 ${ }^{2}$, 李 苓 ${ }^{2}$, 曹晓燕 ${ }^{1,2,3}$
}

(中国海洋大学 1. 海洋化学理论与工程技术教育部重点实验室/海洋高等研究院; 2. 化学化工学院; 3. 青岛海洋科 学与技术国家实验室 海洋生态与环境科学功能实验室, 青岛 266100)

摘 要: 磷是影响藻类生长的重要营养元素, 它在沉积物界面上的吸附是海洋环境中磷循环的重要环节, 金属氧化 物是吸附磷沉积物的重要活性组分之一。采用批量实验法研究不同结构的氧化铝对无机磷的吸附特征, 分别使用二 段一级动力学方程和 Freundlich 等方程对其动力学曲线和平衡吸附等温线进行定量描述, 并对介质盐度、温度等影 响因素进行研究。结果发现, 无定型氧化铝结晶弱且比表面积大, 该特点使其吸附容量大于 $\gamma-\mathrm{Al}_{2} \mathrm{O}_{3}$, 结合表面酸碱 滴定的结果, 可知吸附也与铝氧化物的表面酸碱性质相关。分析影响因素可知, 与 $\mathrm{NaNO}_{3}$ 介质相比, 氧化铝吸附海 水中的磷受到阻滞, 且随着介质离子强度增大, 吸附量均呈现降低趋势; $\mathrm{pH}$ 显著影响磷的吸附量, 在 $\mathrm{pH}=5$ 左右, 两种氧化铝表面对磷酸根的吸附达到最大值; 温度升高, 有利于吸附的进行, 该吸附为吸热、自发、熵增加的过程, 两种结构氧化铝的吸附热力学参数无显著差异。

关 键 词: 富营养化; 磷; 铝氧化物; 结构; 吸附

中图分类号: X703 文献标识码: A

\section{Phosphorus Sorption Characteristics on Aluminum Oxides with Different Structures}

\author{
LI Jing ${ }^{1,2}$, LIU Xiaoyue ${ }^{2}$, QIU Qianfeng ${ }^{2}$, LI Ling ${ }^{2}$, CAO Xiaoyan ${ }^{1,2,3}$
}

(1. Key Laboratory of Marine Chemistry Theory and Technology, Ministry of Education /Institute for Advanced Ocean Study, Ocean University of China, Qingdao 266100, China; 2. College of Chemistry and Chemical Engineering, Ocean University of China, Qingdao 266100, China; 3. Laboratory for Marine Ecology and Environmental Science, Qingdao National Laboratory for Marine Science and Technology, Qingdao 266100, China)

\begin{abstract}
Phosphorus is an important nutrient element that affects the growth of algae. The sorption of phosphorus on the sediments plays an important role in its bio-geochemical cycle, with metal oxides such as alumina being one of the important active components for the process. The sorption behavior of phosphorus on two kinds of alumina $\left(\gamma-\mathrm{Al}_{2} \mathrm{O}_{3}\right.$, amorphous alumina) was studied through batch methods. Both kinetics and thermodynamics of the process were investigated, as well as the effects of temperature, salinity, and $\mathrm{pH}$ of the medium on the process. The sorption kinetic curves could be described by a two-compartment first order equation, and the isotherms fit Freundlich equation well. The amorphous alumina has a larger specific surface area, and its sorption ability is stronger than that of $\gamma-\mathrm{Al}_{2} \mathrm{O}_{3}$. Based on the results of surface acid-base titration, the sorption behavior is also considered to be related to the surface acidity and alkalinity of the aluminum oxides. Compared with $\mathrm{NaNO}_{3}$ medium, the sorption of phosphorus in seawater was weakened. The sorption capacity decreases with the increase of the ionic strength. pH significantly affected the
\end{abstract}

收稿日期: 2019-08-28; 收到修改稿日期：2020-01-13

基金项目：国家自然科学基金(41676064); 国家重点研发计划项目(2016YFA0601301)

National Natural Science Foundation of China (41676064); National Key Research and Development Program of China (2016YFA0601301)

作者简介: 李 敬(1995-), 女, 硕士研究生. E-mail: 840609162@qq.com LI Jing(1995-), female, Master candidate. E-mail: 840609162@qq.com

通讯作者: 曹晓燕，教授, E-mail: caoxy@ouc.edu.cn CAO Xiaoyan, professor. E-mail: caoxy@ouc.edu.cn 
sorption ability of the oxides with the maximum capacity at $\mathrm{pH}=5$. Higher temperatures are favorable to the sorption progress, which is endothermic and spontaneous with entropy increasing. The difference in the thermodynamic parameters of the two alumina seemed unremarkable.

Key words: eutrophication; phosphorus; aluminum oxides; structure; sorption

磷是影响藻类生长, 造成水体富营养化的重要 元素之一, 而沉积物是海洋中氮、磷等营养盐蓄积 和释放的重要场所, 它对水体中磷起着重要调节作 用 ${ }^{[1-3]}$ 。天然沉积物吸附活性组分主要包括碳酸盐、 粘土矿物、有机质、活泼金属氧化物等, 其中氧化 铝包括水合氧化铝矿物是在天然水体沉积物中普遍 存在的金属氧化物之一 ${ }^{[4]}$ 。已有研究表明, 沉积物吸 附释放磷的过程会受到沉积物中无定形铁和氧化铝 的影响, 氧化铝发挥的作用不容小䖍 ${ }^{[5-7]}$ 。另外, 铝 氧化物以其吸附性能优良、工艺简单、原料廉价、 纯度高等优点, 作为吸附剂处理水体中磷污染取得 了较好的效果, 并引起了人们的关注 ${ }^{[8-9]}$ 。

沉积物中铝氧化物多以三水铝石、三羟铝石、 勃姆石和水铝石的形式存在, 它们的晶体结构各不 相同 ${ }^{[6]} 。 \gamma-\mathrm{Al}_{2} \mathrm{O}_{3}$ 是一种常见的结晶态氧化铝, 表面 有特殊的活性位点, 粒径小、结晶强、吸附能力强, 化学反应活性高, 常作为吸附剂应用于污染物吸附 处理, 亦常用于研究污染物在固-液界面化学上的 吸附机制 ${ }^{[10-12]}$ 。无定形(氢)氧化铝内部原子结构长 程无序、结晶弱、比表面积大、化学活性位点多, 存 在于土壤或沉积物中对磷的吸着固定起着重要作 用。鉴于两者广泛存在于沉积物中且在结晶程度上 存在明显差异, 本工作拟合成 $\gamma-\mathrm{Al}_{2} \mathrm{O}_{3}$ 与无定形氧 化铝, 考察铝氧化物晶体结构对吸附磷行为的影 响。同时由于目前关于铝氧化物吸附磷的研究大都 集中于高浓度磷污水介质 ${ }^{[13]}$, 研究介质多采用 $\mathrm{NaCl} 、 \mathrm{KCl} 、 \mathrm{NaNO}_{3}$ 溶液等模拟体系 ${ }^{[12-15]}$ ，与自然 水系中磷的存在情况相差甚远，本研究将使用天然 海水(NSW)介质来模拟实际环境, 在较低初始磷浓 度下探究了两种铝氧化物对吸附磷的行为, 并探究 了介质盐度和温度等条件对吸附热力学过程的影响, 为进一步探讨沉积物活性组分对吸附磷的作用提供 数据参考。

\section{1 实验方法}

\section{1 氧化铝制备}

参考文献[16]制备 $\gamma-\mathrm{Al}_{2} \mathrm{O}_{3}$ 和无定形氧化铝，具 体过程如下。

1) $\gamma-\mathrm{Al}_{2} \mathrm{O}_{3}$ 制备: 烧瓶中加入 $72.42 \mathrm{~g} \mathrm{AlCl}_{3} \cdot 6 \mathrm{H}_{2} \mathrm{O}$, $9.0 \mathrm{~g}$ 聚乙二醇( PEG 6000), $240 \mathrm{~mL}$ 去离子水, 搅拌
均匀后在油浴中升温至 $80{ }^{\circ} \mathrm{C}$, 用 $\mathrm{KOH}$ 溶液调节至 $\mathrm{pH}=7$, 继续摚拌后陈化 $24 \mathrm{~h}$, 离心分离, 沉淀清洗 后在 $110{ }^{\circ} \mathrm{C}$ 干燥 $16 \mathrm{~h}$, 再在 $520{ }^{\circ} \mathrm{C}$ 煅烧 $4 \mathrm{~h}$ 得到 $\gamma-\mathrm{Al}_{2} \mathrm{O}_{3}$ ，研磨备用。

2)无定形氧化铝制备: 烧瓶中加入 $72.40 \mathrm{~g}$ $\mathrm{Al}_{2} \mathrm{Cl}_{3} \cdot 6 \mathrm{H}_{2} \mathrm{O}, 9.0 \mathrm{~g}$ 聚乙二醇, $240 \mathrm{~mL}$ 去离子水，搅拌 均匀。在油浴 $80{ }^{\circ} \mathrm{C}$ 中边搅拌边滴加 $25 \mathrm{wt} \%$ 28wt $\%$ 的氨水至体系 $\mathrm{pH}=7$, 继续摚拌后陈化 $24 \mathrm{~h}$, 离心并 洗涤后在 $45{ }^{\circ} \mathrm{C}$ 烘箱中放置 $30 \mathrm{~h}$, 然后在 $370{ }^{\circ} \mathrm{C}$ 煅 烧 $6 \mathrm{~h}$ 得到无定形氧化铝, 研磨备用。

\section{2 样品表征}

利用 $\mathrm{X}$ 射线粉末衍射仪(D8 ADVANCE)对两种 铝氧化物的晶型进行分析, 并利用 FT-IR 红外光谱 仪(尼高力 380)对样品结构进行表征。利用冷场发射 扫描电子显微镜(日立 S4800)观察样品表面形貌, 用 全自动比表面分析仪(Micromeritic ASAP2020M+C) 测试样品的比表面积等。利用瑞士万通 809 自动电 位滴定仪和碱返滴定法测试表征铝氧化物的表面酸 碱性质。

\section{3 无机磷吸附实验}

采用批量取样法测定铝氧化物样品上磷的吸附 动力学曲线和吸附等温线。

称取适量铝氧化物样品放入雉形瓶中, 加入 $50 \mathrm{~mL}$ 一定浓度的磷酸盐溶液。在 $(25 \pm 1){ }^{\circ} \mathrm{C}$ 、振速 为 $150 \mathrm{r} \cdot \mathrm{min}^{-1}$ 的恒温水浴振荡器中振荡, 定时取样, 离心分离上清液并过滤, 采用磷钼蓝法测定其中磷 浓度。以时间 $t$-磷吸附量 $Q_{t}$ 为横纵坐标, 绘制吸附 动力学曲线, 并对比磷酸盐在天然海水 (NSW) 和 $0.7 \mathrm{~mol} \cdot \mathrm{L}^{-1} \mathrm{NaNO}_{3}$ 介质中在氧化铝上的吸附动力学 差异。

称量适量铝氧化物放入雉形瓶中, 分别加入 $50 \mathrm{~mL}$ 一系列不同浓度的磷酸盐溶液。在 $25{ }^{\circ} \mathrm{C}$ 振 荡器中振荡 $24 \mathrm{~h}$, 其他过程及测定方法同上。以平 衡浓度 $C_{\mathrm{e}}$ 和磷的平衡吸附量 $Q_{\mathrm{e}}$ 为横纵坐标, 绘制 吸附热力学曲线。

在浓度分别为 $0.01 、 0.10$ 和 $0.70 \mathrm{~mol} \cdot \mathrm{L}^{-1}$ 的 $\mathrm{NaNO}_{3}$ 介质中进行铝氧化物对磷的等温吸附实验, 考察介 质离子强度对吸附过程的影响; 在以 $0.7 \mathrm{~mol} \cdot \mathrm{L}^{-1}$ $\mathrm{NaNO}_{3}$ 为背景电解质的磷酸盐溶液中, 用稀 $\mathrm{HNO}_{3}$ 或稀 $\mathrm{NaOH}$ 将 $\mathrm{pH}$ 分别调至不同值, 其他过程同等 温吸附实验, 测试铝氧化物对磷的 $\mathrm{pH}$ 吸附边曲线。 
实验所用试剂均为分析纯; 天然海水(NSW)取 自青岛近海, 陈化一个月以上使其含有的磷可以忽 略不计 ${ }^{[17]}$, 用 $0.45 \mu \mathrm{m}$ 的滤膜过滤备用。

\section{2 结果与讨论}

\section{1 样品结构及表面性质}

\subsubsection{X 射线衍射与红外分析}

图 1 为 $\gamma-\mathrm{Al}_{2} \mathrm{O}_{3}$ 样品的 XRD 图谱, 其衍射峰位置 以及峰的相对强度与 $\gamma-\mathrm{Al}_{2} \mathrm{O}_{3}$ (JCPDS 10-0425)相匹 配, 说明实验制备的样品为晶型比较完整的 $\gamma-\mathrm{Al}_{2} \mathrm{O}_{3}$ 颗粒。从图 1 还可以看出, 制备的无定型氧化铝衍 射峰吸收强度较小, 与 $\gamma-\mathrm{Al}_{2} \mathrm{O}_{3}$ 相比其衍射峰更宽, 可见其结晶度较差。

图 2 为两种氧化铝的傅里叶红外光谱图。 $\mathrm{Al}_{2} \mathrm{O}_{3}$ 的吸收峰位于 $3300,3093,1075,746,618 \mathrm{~cm}^{-1[18]}$, $3000 \mathrm{~cm}^{-1}$ 以上的吸收峰归属于 $\mathrm{AlO}-\mathrm{H}$ 键的伸缩振 动峰, $1075 \mathrm{~cm}^{-1}$ 处的吸收峰归属于 $\mathrm{AlO}-\mathrm{H}$ 键的强的 弯曲振动峰。

\subsection{2 表面性质表征}

两种氧化铝的表观形貌如图 3 所示, 可以清晰 看出: $\gamma-\mathrm{Al}_{2} \mathrm{O}_{3}$ 呈长柱状, 形态分布较规则, 而无定 型氧化铝形态不规则, 多呈不规则片状及疏松多孔 絮状等, 结晶度差。

利用自动表面和孔径分析仪, 基于 $\mathrm{N}_{2}$ 吸脱附法 测定了两种氧化铝颗粒的比表面积, 其中 $\gamma-\mathrm{Al}_{2} \mathrm{O}_{3}$ 的比表面积为 $195.43 \mathrm{~m}^{2} \cdot \mathrm{g}^{-1}$, 无定形氧化铝的比表 面积为 $297.13 \mathrm{~m}^{2} \cdot \mathrm{g}^{-1}$, 无定形氧化铝比表面积明显 大于 $\gamma-\mathrm{Al}_{2} \mathrm{O}_{3}$ 。

利用瑞士万通 809 全自动电位滴定仪, 采用碱 返滴定方法, 拟合计算得出不同离子强度介质中氧 化铝表面的表面羟基位浓度 $H_{\mathrm{s}}$ 、零净质子电荷点 $\mathrm{pH}_{\mathrm{PZNPC}}$ 、总反应 $\mathrm{H}^{+}$浓度 $(\mathrm{TOTH})$ 等参数, 结果列于 表 1 。由表可知, 随着介质离子强度增大, $\gamma-\mathrm{Al}_{2} \mathrm{O}_{3}$ 和 无定形氧化铝的表面羟基位浓度 $H_{\mathrm{s}}$ 均减小, 说明离 子强度增大阻碍了氧化铝的表面羟基化，且 $\gamma-\mathrm{Al}_{2} \mathrm{O}_{3}$ 的 $H_{\mathrm{s}}$ 稍大于无定形氧化铝。由于天然海水是 $\mathrm{pH}=8$ 的缓冲体系, 且 $0.7 \mathrm{~mol} \cdot \mathrm{L}^{-1}$ 的 $\mathrm{NaNO}_{3}$ 介质盐度与天
然海水相近, 所以此处只对比 $\mathrm{pH}=8$ 时 $0.7 \mathrm{~mol} \cdot \mathrm{L}^{-1}$ 的 $\mathrm{NaNO}_{3}$ 介质中的表面总反应 $\mathrm{H}^{+}$浓度(TOTH)值, $\gamma-\mathrm{Al}_{2} \mathrm{O}_{3}$ 和无定形氧化铝的 TOTH 值分别为 -0.809 和 $-1.380 \mathrm{mmol} \cdot \mathrm{L}^{-1}$, 可见无定形氧化铝的表面去质 子化更显著; 本实验中, $\gamma-\mathrm{Al}_{2} \mathrm{O}_{3}$ 和无定形氧化铝的 零净质子电荷点 $\mathrm{pH}_{\mathrm{PZNPC}}$ 均在酸性范围内, 它们的 $\mathrm{pH}_{\mathrm{PZNPC}}$ 分别为 4.52 和 4.25 。

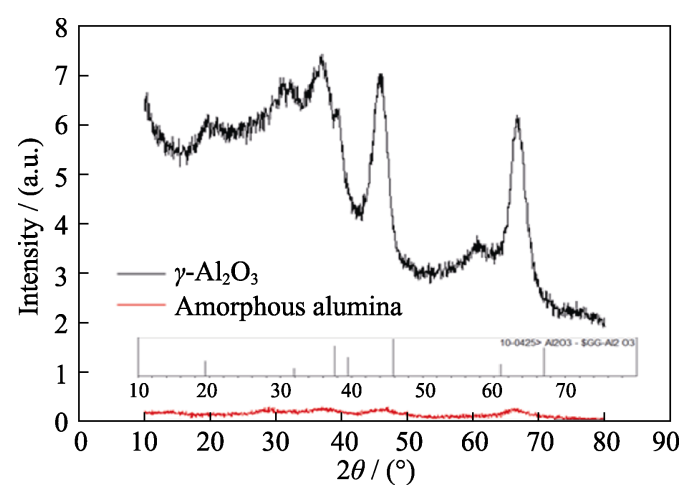

图 $1 \gamma-\mathrm{Al}_{2} \mathrm{O}_{3}$ 、无定形氧化铝的 XRD 图谱

Fig. 1 XRD patterns of $\gamma-\mathrm{Al}_{2} \mathrm{O}_{3}$ and amorphous alumina

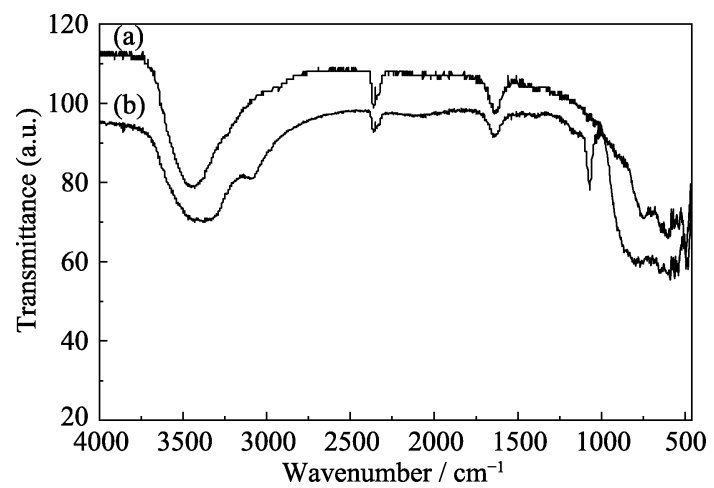

图 $2 \gamma-\mathrm{Al}_{2} \mathrm{O}_{3}$ (a)、无定形氧化铝(b)的傅里叶红外光谱图

Fig. 2 Fourier infrared spectra of $\gamma-\mathrm{Al}_{2} \mathrm{O}_{3}$ (a) and amorphous alumina (b)
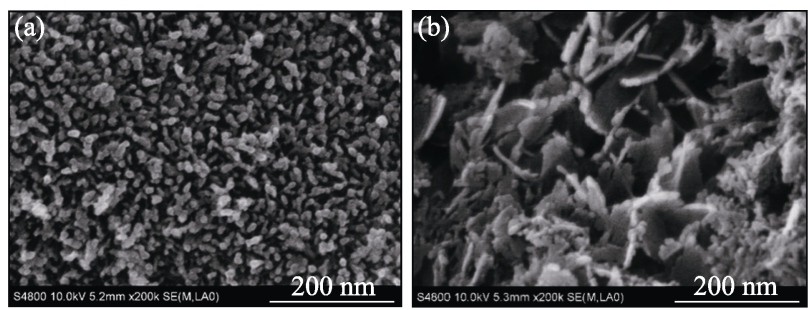

图 $3 \gamma-\mathrm{Al}_{2} \mathrm{O}_{3}$ (a) 和无定型氧化铝(b)的 SEM 照片

Fig. 3 SEM images of $\gamma-\mathrm{Al}_{2} \mathrm{O}_{3}$ (a) and amorphous alumina (b)

表 1 不同离子强度介质中两种铝氧化物的表面酸碱性质

Table 1 Surface acid-base properties of two aluminum oxides in different ionic strength media

\begin{tabular}{|c|c|c|c|c|c|c|}
\hline \multirow{2}{*}{$\begin{array}{c}\mathrm{NaNO}_{3} \text { concentration/ } \\
\left(\mathrm{mol} \cdot \mathrm{L}^{-1}\right)\end{array}$} & \multicolumn{2}{|c|}{$H_{\mathrm{S}}$ concentration $/\left(\mathrm{mol} \cdot \mathrm{L}^{-1}\right)$} & \multicolumn{2}{|c|}{$\mathrm{TOTH}_{\mathrm{pH}=8} /\left(\mathrm{mmol} \cdot \mathrm{L}^{-1}\right)$} & \multicolumn{2}{|r|}{$\mathrm{pH}_{\mathrm{PZNPC}}{ }^{*}$} \\
\hline & $\gamma-\mathrm{Al}_{2} \mathrm{O}_{3}$ & Amorphous alumina & $\gamma-\mathrm{Al}_{2} \mathrm{O}_{3}$ & Amorphous alumina & $\gamma-\mathrm{Al}_{2} \mathrm{O}_{3}$ & Amorphous alumina \\
\hline 0.01 & $3.35 \times 10^{-3}$ & $2.69 \times 10^{-3}$ & - & - & & \\
\hline 0.10 & $2.94 \times 10^{-3}$ & $1.79 \times 10^{-3}$ & - & - & 4.52 & 4.25 \\
\hline 0.70 & $2.75 \times 10^{-3}$ & $1.09 \times 10^{-3}$ & -0.809 & -1.380 & & \\
\hline
\end{tabular}

*Note: the value is tested based on the three ion strength media 


\section{2 不同介质中的吸附动力学}

对比表面较大的无定型氧化铝进行吸附动力学 曲线测试, 以确定吸附平衡时间。初始磷的浓度为 $2 \mathrm{mg} \cdot \mathrm{L}^{-1}$, 选择天然海水 $(\mathrm{NSW})$ 以及离子强度相近 的 $0.7 \mathrm{~mol} \cdot \mathrm{L}^{-1} \mathrm{NaNO}_{3}$ 溶液为吸附介质, 固液比为 $0.08 \mathrm{~g} \cdot \mathrm{L}^{-1}$, 结果如图 4 所示。吸附 $5 \mathrm{~h}$ 内, 无定型氧 化铝对无机磷呈现快速吸附; 吸附 $5 \mathrm{~h}$ 后, 吸附量随 着吸附时间的延长变化减缓; 吸附 $20 \mathrm{~h}$ 后, 吸附趋 于平衡状态。为保证热力学吸附充分进行, 平衡吸 附时间选定为 $24 \mathrm{~h}$ 。

采用两段一级动力学方程 (1) ${ }^{[19]}$ 对吸附过程进 行拟合，结果见表 2 。海水介质中，无定型氧化铝吸 附磷的快吸附速率常数 $k_{\text {rap }}$ 、慢吸附速率常数 $k_{\text {slow }}$ 以及平衡吸附量 $Q_{\mathrm{e}}$ 值均小于在 $0.7 \mathrm{~mol} \cdot \mathrm{L}^{-1} \mathrm{NaNO}_{3}$ 介质中的值, 可知天然海水介质抑制了磷在氧化铝 上的吸附，这是由于天然海水中复杂的离子所产生 的竞争作用导致。 $F_{\text {rap }}$ 表示以物理吸附为主的快吸附 部分所占分数, $F_{\text {slow }}$ 表示以化学吸附为主的慢吸附 部分所占分数, 对比可知, 相较于 $\mathrm{NaNO}_{3}$ 介质, 天 然海水中无机磷的物理吸附作用减小, 化学吸附作 用增强，但整体仍以物理吸附作用为主。

$$
\frac{Q_{\mathrm{e}}-Q_{\mathrm{t}}}{Q_{\mathrm{e}}}=F_{\text {rap }} \exp \left(-k_{\text {rap }} t\right)+F_{\text {slow }} \exp \left(-k_{\text {slow }} t\right)
$$

\section{3 不同离子强度介质中的吸附等温线}

图 5 是不同离子强度 $\mathrm{NaNO}_{3}$ 介质中磷在两种氧 化铝上的等温吸附曲线, 实验固液比 $0.08 \mathrm{~g} \cdot \mathrm{L}^{-1}$, 初 始磷浓度 $\left(\gamma-\mathrm{Al}_{2} \mathrm{O}_{3}\right.$ : $0 \sim 2 \mathrm{mg} \cdot \mathrm{L}^{-1}$; 无定型氧化铝：0 $5 \mathrm{mg} \cdot \mathrm{L}^{-1}$ )。分别用 Langmuir(公式 2) 和 Freundlich(公 式 3)吸附方程对实验数据进行拟合，拟合参数见表 3, 对比 $r^{2}$ 可知 Freundlich 方程更适合描述该过程。在 各种不同离子强度介质中, 无定型氧化铝对无机磷的 吸附量均明显大于 $\gamma-\mathrm{Al}_{2} \mathrm{O}_{3}, K_{\mathrm{F}}$ 值约是 $\gamma-\mathrm{Al}_{2} \mathrm{O}_{3}$ 的 2 倍, 也说明无定型氧化铝对无机磷的吸附能力较强, 这 与无定型氧化铝疏松多孔的无定形结构及较大的比

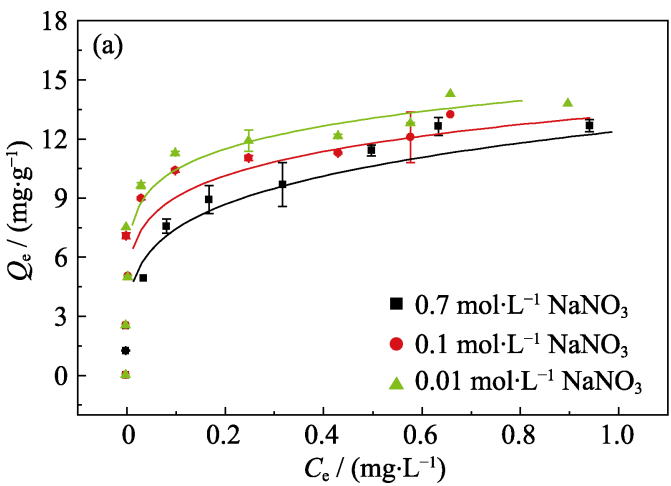

表面积有关。随着介质离子强度的增大, 两种氧化铝 上的 $K_{\mathrm{F}}$ 均有所减小, 其中 $\gamma-\mathrm{Al}_{2} \mathrm{O}_{3}$ 的吸附能力变化更 为显著, 说明离子强度增大, 不利于 $\gamma-\mathrm{Al}_{2} \mathrm{O}_{3}$ 和无定型 氧化铝对无机磷的吸附，可能是因为介质中的离子 增多会与磷酸根竞争氧化物表面的吸附位，从而导致 吸附能力减小, 同时沉积物表面上 $H_{\mathrm{s}}$ 的减小, 也在一 定程度上减少了荷负电的磷酸根在沉积物上的吸附。

$$
\begin{gathered}
Q_{\mathrm{e}}=K_{\mathrm{F}} C_{\mathrm{e}}^{1 / n} \\
Q_{\mathrm{e}}=\frac{Q_{\mathrm{m}} K_{\mathrm{L}} C_{\mathrm{e}}}{1+K_{\mathrm{L}} C_{\mathrm{e}}}
\end{gathered}
$$

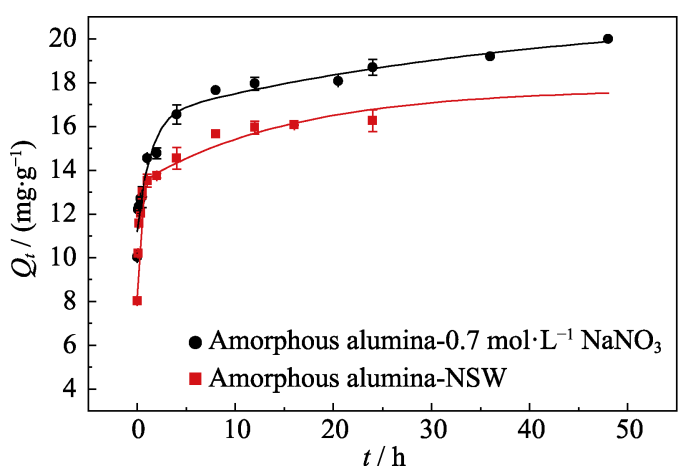

图 4 无定形氧化铝对磷酸盐的吸附动力学及拟合曲线 Fig. 4 Sorption kinetics curves of phosphate on amorphous alumina

Dot: experimental data; Line:two-compartment first-order model fitting

表 2 磷酸盐在无定形氧化铝上的吸附动力学参数

Table 2 Sorption kinetic parameters of phosphorus on amorphous alumina

\begin{tabular}{ccccccc}
\hline Medium & $F_{\text {rap }}$ & $F_{\text {slow }}$ & $k_{\text {slow }} / \mathrm{h}^{-1}$ & $k_{\text {rap }} / \mathrm{h}^{-1}$ & $Q_{\mathrm{e}} /\left(\mathrm{mg} \cdot \mathrm{g}^{-1}\right)$ & $r^{2}$ \\
\hline $0.7 \mathrm{~mol} \cdot \mathrm{L}^{-1}$ & 0.813 & 0.187 & 0.977 & 26.32 & 21.42 & 0.977 \\
$\mathrm{NaNO}_{3}$ & 0.97 & & & & & \\
$\mathrm{NSW}$ & 0.740 & 0.260 & 0.193 & 20.23 & 16.56 & 0.988 \\
\hline
\end{tabular}

${ }^{\mathrm{a}} Q_{\mathrm{e}}$ and $Q_{t}(\mathrm{mg} / \mathrm{g})$ are the sorption amounts of sorbate at equilibrium and various time $t(\mathrm{~min}), F_{\text {rap }}$ and $F_{\text {slow }}$ are the rapid and slow fractions of the process $\left(F_{\text {rap }}+F_{\text {slow }}=1\right)$, and $k_{\text {rap }}$ and $k_{\text {slow }}$ are the rate constants of the rapid and slow sorption

图 5 在不同离子强度 $\mathrm{NaNO}_{3}$ 介质中, 磷酸盐在 $\gamma-\mathrm{Al}_{2} \mathrm{O}_{3}$ (a) 和无定形氧化铝(b)上的等温吸附曲线

Fig. 5 Sorption isotherms of phosphate on $\gamma-\mathrm{Al}_{2} \mathrm{O}_{3}$ (a) and amorphous alumina (b) in $\mathrm{NaNO}_{3}$ with different concentrations Dot: experimental data; Line: Freundlich model fitting 
表 3 不同离子强度介质中磷酸盐在 $\gamma-\mathrm{Al}_{2} \mathrm{O}_{3}$ 和无定形氧化铝上的等温吸附参数

Table 3 Isothermal sorption parameters of phosphates on $\gamma-\mathrm{Al}_{2} \mathrm{O}_{3}$ and amorphous alumina

\begin{tabular}{|c|c|c|c|c|c|c|c|}
\hline \multirow{2}{*}{ Sorbent } & \multirow{2}{*}{$I /\left(\mathrm{mol} \cdot \mathrm{L}^{-1}\right)$} & \multicolumn{3}{|c|}{ Freundlich } & \multicolumn{3}{|c|}{ Langmuir } \\
\hline & & $K_{\mathrm{F}} /\left(\mathrm{mg} \cdot \mathrm{g}^{-1}\right)\left(\mathrm{L} \cdot \mathrm{mg}^{-1}\right)^{1 / n}$ & $n$ & $r^{2}$ & $Q_{\mathrm{m}} /\left(\mathrm{mg} \cdot \mathrm{g}^{-1}\right)$ & $K_{\mathrm{L}} /\left(\mathrm{L} \cdot \mathrm{mg}^{-1}\right)$ & $r^{2}$ \\
\hline \multirow{3}{*}{$\gamma-\mathrm{Al}_{2} \mathrm{O}_{3}$} & 0.7 & 12.39 & 4.52 & 0.942 & 14.93 & 5.288 & 0.848 \\
\hline & 0.1 & 13.20 & 6.08 & 0.912 & 13.47 & 18.29 & 0.702 \\
\hline & 0.01 & 14.37 & 7.20 & 0.896 & 14.28 & 27.90 & 0.485 \\
\hline \multirow{3}{*}{ Amorphous alumina } & 0.7 & 26.28 & 3.27 & 0.933 & 34.70 & 3.828 & 0.838 \\
\hline & 0.1 & 27.66 & 4.09 & 0.933 & 34.60 & 4.888 & 0.820 \\
\hline & 0.01 & 28.36 & 3.24 & 0.907 & 43.22 & 2.046 & 0.817 \\
\hline
\end{tabular}

${ }^{\mathrm{a}} Q_{\mathrm{m}}$ are the maximum amount of phosphorous sorbed onto sorbent $(\mathrm{mg} / \mathrm{g}) ; K_{\mathrm{L}}$ is the Langmuir sorption coefficient $(\mathrm{L} / \mathrm{mg}) ; K_{\mathrm{F}}$ and $n$ are the Freundlich constants which relate to sorption capacity and sorption intensity; $I$ is ionic strength of medium

\section{4 温度对吸附等温线的影响}

在 $0.7 \mathrm{~mol} \cdot \mathrm{L}^{-1} \mathrm{NaNO}_{3}$ 介质中, 不同温度下磷酸 盐在两种氧化铝样品表面的吸附结果如图 6 所示。 由图可见随着温度升高, 两种类型氧化铝样品对磷 的吸附量均呈增加趋势, 表明氧化铝对磷的吸附是 吸热过程。吸附平衡常数以 Freundlich 吸附系数计, 利用范霍夫方程获得吸附过程的热力学参数, 结果 见表 4 。从表 4 可以发现, 两种结构的氧化铝在吸附 磷过程中的标准焓变、标准吉布斯自由能及熵变等 无显著差异。吸附过程中 $\Delta H^{\theta}>0, \Delta G^{\theta}<0, \Delta S^{\theta}>0$ 说明 整个吸附过程是吸热、自发、熵增加的过程。磷酸 盐在 $\gamma-\mathrm{Al}_{2} \mathrm{O}_{3}$ 上的吸附 $\Delta G^{\theta}$ 在 $-24.26 \sim-23.18 \mathrm{~kJ} \cdot \mathrm{mol}^{-1}$ 之间，在无定形氧化铝上的吸附 $\Delta G^{\theta}$ 在-26.46 $-25.21 \mathrm{~kJ} \cdot \mathrm{mol}^{-1}$ 之间, 表明两种氧化铝对磷的吸附

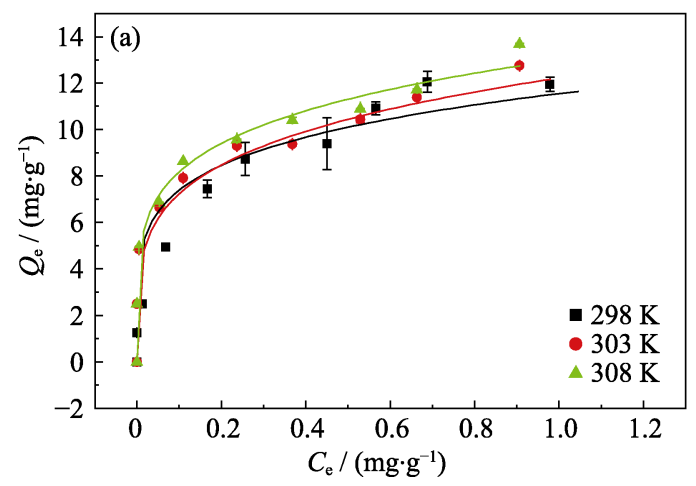

均是物理吸附和化学吸附的共同结果，这与李显波 等 ${ }^{[20]}$ 对活性氧化铝的研究结果相一致。

\section{5 铝氧化物吸附磷的 $\mathrm{pH}$ 边曲线}

图 7 为磷酸盐在 $\gamma-\mathrm{Al}_{2} \mathrm{O}_{3}$ 和无定形氧化铝上的吸 附 $\mathrm{pH}$ 边曲线。由图 7 可知, 无定形氧化铝和 $\gamma-\mathrm{Al}_{2} \mathrm{O}_{3}$ 样品的吸附量随 $\mathrm{pH}$ 的变化趋势与幅度相似，当 $\mathrm{pH}$ 为 5 时, 对磷的吸附量呈现最大值, 分别达到 9.880 和 $20.49 \mathrm{mg} \cdot \mathrm{g}^{-1}$ 。在实验 $\mathrm{pH}$ 范围内无定形氧化铝对 磷的吸附量均比 $\gamma-\mathrm{Al}_{2} \mathrm{O}_{3}$ 的吸附量高。

$\mathrm{pH}$ 对磷酸根在铝氧化物上吸附的影响可以从 吸附剂的表面性质和磷酸盐的形态分布两方面来分 析。由表 1 可知, $\gamma-\mathrm{Al}_{2} \mathrm{O}_{3}$ 、无定形氧化铝的零净质 子电荷点 $\mathrm{pH}$ PZNPC 分别为 4.52 和 4.25 ; 磷酸根在水 溶液中的形态分布可以根据其解离常数 $\left(\mathrm{p} K_{\mathrm{a} 1}=2.15\right.$,

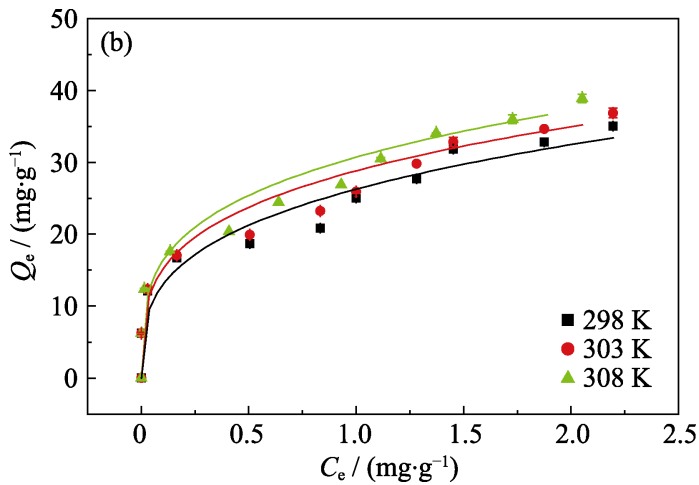

图 6 不同温度下, 磷酸盐在 $\gamma-\mathrm{Al}_{2} \mathrm{O}_{3}(\mathrm{a})$ 和无定形氧化铝(b)上的等温吸附曲线

Fig. 6 Sorption isotherms of phosphate on $\gamma-\mathrm{Al}_{2} \mathrm{O}_{3}(\mathrm{a})$ and amorphous alumina (b) at different femperatures Dot: experimental data; Line: Freundlich model fitting

表 4 磷酸盐在 $\gamma-\mathrm{Al}_{2} \mathrm{O}_{3}$ 和无定形氧化铝的吸附热力学参数

Table 4 Sorption thermodynamic parameters of phosphate on $\gamma-\mathrm{Al}_{2} \mathrm{O}_{3}$ and amorphous alumina

\begin{tabular}{cccccccc}
\hline Adsorbent & $T / \mathrm{K}$ & $K_{\mathrm{F}} /\left(\mathrm{mg} \cdot \mathrm{g}^{-1}\right)\left(\mathrm{L} \cdot \mathrm{mg}^{-1}\right)^{1 / n}$ & $n$ & $r^{2}$ & $\Delta G^{\theta} /\left(\mathrm{kJ} \cdot \mathrm{mol}^{-1}\right)$ & $\Delta H^{\theta} /\left(\mathrm{kJ} \cdot \mathrm{mol}^{-1}\right)$ & $\Delta S^{\theta} /\left(\mathrm{J} \cdot \mathrm{mol}^{-1} \cdot \mathrm{K}^{-1}\right)$ \\
\hline \multirow{2}{*}{$\gamma-\mathrm{Al}_{2} \mathrm{O}_{3}$} & 298 & 11.55 & 5.18 & 0.938 & -23.18 & & \\
& 303 & 12.22 & 4.40 & 0.948 & -23.71 & 9.007 & 108.0 \\
\hline \multirow{3}{*}{ Amorphous alumina } & 308 & 12.99 & 4.98 & 0.952 & -24.26 & & \multirow{2}{*}{124.8} \\
& 298 & 26.28 & 3.27 & 0.933 & -25.21 & & \\
\hline
\end{tabular}

${ }^{a}$ Standard free Gibs energy change $\left(\Delta G^{\theta}\right)$, standard enthalpy change $\left(\Delta H^{\theta}\right)$, and standard entropy change $\left(\Delta S^{\theta}\right)$ 


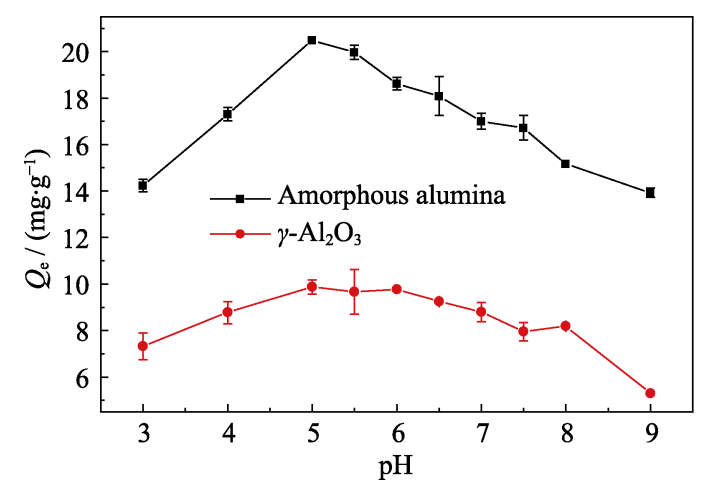

图 7 磷酸盐在 $\gamma-\mathrm{Al}_{2} \mathrm{O}_{3}$ 和无定形氧化铝上的吸附 $\mathrm{pH}$ 边曲线 Fig. 7 Sorption $\mathrm{pH}$ edges curves of $\mathrm{P}$ on $\gamma-\mathrm{Al}_{2} \mathrm{O}_{3}$ and amorphous alumina

$\left.\mathrm{p} K_{\mathrm{a} 2}=9.35, \mathrm{p} K_{\mathrm{a} 3}=21.7\right)$ 计算得出，在 $\mathrm{pH} 3 \sim 6$ 范围内， 以 $\mathrm{H}_{2} \mathrm{PO}_{4}^{-}$的形态存在。当 $\mathrm{pH}$ 小于 5 时, 铝氧化物 表面荷正电, 有利于吸附带负电荷的 $\mathrm{H}_{2} \mathrm{PO}_{4}^{-}$, 但在 $\mathrm{pH}$ 由 5 降到 3 的过程中, 铝氧化物可能存在的溶解 导致溶出的铝离子与磷酸根离子形成溶液络合物 ${ }^{\left[{ }^{[}\right]}$, 从而使得表面吸附减少, 因此在 $\mathrm{pH}$ 为 5 时出现吸附 最高值。随着 $\mathrm{pH}$ 升高, 当 $\mathrm{pH}$ 大于 5 时, 氧化铝表 面荷负电, 且当 $\mathrm{pH}$ 大于 7 时, 磷酸根离子以 $\mathrm{HPO}_{4}{ }^{2-}$ 的形式存在 ${ }^{[21]}$ 。Chubar 等 ${ }^{[22]}$ 研究认为 $\mathrm{HPO}_{4}{ }^{2-}$ 形态 比 $\mathrm{H}_{2} \mathrm{PO}_{4}{ }^{-}$形态更难于与金属氧化物表面羟基发生 配体交换反应, 所以此条件不利于吸附的进行, 故 而吸附量呈降低趋势。

\section{3 结论}

1)氧化铝对磷的吸附动力学过程分为快慢吸附 两个部分, 平衡时间为 $24 \mathrm{~h}$, 可用两段一级动力学方 程描述。相对于 $\mathrm{NaNO}_{3}$ 介质, 天然海水中磷在氧化 铝上的平衡吸附量及吸附速率均降低，同时吸附过 程中的物理作用减小，化学作用增加，吸附过程仍 以物理吸附作用为主。

2)氧化铝的吸附等温线符合 Freundlich 方程。无 定型氧化铝对磷的吸附能力大于 $\gamma-\mathrm{Al}_{2} \mathrm{O}_{3}$ 。吸附过程 中 $\Delta H^{\theta}>0, \Delta G^{\theta}<0, \Delta S^{\theta}>0$, 两种氧化铝的吸附热力学 参数无明显差异, 整个吸附过程是吸热、自发、熵增 加的过程。

3)离子强度增大不利于无机磷在氧化铝上的吸 附, 吸附系数 $K_{\mathrm{F}}$ 呈下降趋势, 其中 $\gamma-\mathrm{Al}_{2} \mathrm{O}_{3}$ 的吸附能力 变化更为显著。 $\mathrm{pH}$ 对磷在无定形氧化铝和 $\gamma-\mathrm{Al}_{2} \mathrm{O}_{3}$ 上的吸附行为有类似的变化趋势与幅度, $\mathrm{pH}$ 边曲线 均呈倒“U”型, 均在 $\mathrm{pH}$ 为 5 时对磷的吸附量最大。

\section{参考文献:}

[1] JALALI M, PEIKAM E N. Phosphorus sorption-desorption behaviour of river bed sediments in the Abshineh river, Hamedan,
Iran, related to their composition. Environmental Monitoring and Assessment, 2013, 185(1): 537-552.

[2] COTOVICZ JUNIOR L C, MACHADO E D, BRANDINI N, et al. Distributions of total, inorganic and organic phosphorus in surface and recent sediments of the sub-tropical and semi-pristine Guaratuba Bay estuary, SE Brazil. Environmental Earth Sciences, 2014, 72(2): 373-386.

[3] HUANG S, HUANG H, ZHU H. Effects of the addition of iron and aluminum salt on phosphorus adsorption in wetland sediment. Environmental Science and Pollution Research, 2016, 23(10): 10022-10027.

[4] QIAN L, MA M, CHENG D. The effect of water chemistry on adsorption and desorption of $\mathrm{U}(\mathrm{VI})$ on nano-alumina. Journal of Molecular Liquids, 2014, 197: 295-300.

[5] CUI Y, XIAO R, XIE Y, et al. Phosphorus fraction and phosphate sorption-release characteristics of the wetland sediments in the Yellow River Delta. Physics and Chemistry of the Earth, 2018, 103: 19-27.

[6] 王赫, 贾永锋, 刘利. 沉积物中典型氧化物矿物吸附的镉对芦 苇的生物有效性研究. 环境科学, 2009, 30(6): 215-220.

[7] LAING G D, RINKLEBE J, VANDECASTEELE B, et al. Trace metal behaviour in estuarine and riverine floodplain soils and sediments: a review. Science of the Total Environment, 2009, 407(13): 3972-3985.

[8] 杨晓芳, 王东升, 孙中溪. 三水铝石 $\left(\gamma-\mathrm{Al}(\mathrm{OH})_{3}\right)$ 和 $\alpha-\mathrm{Al}_{2} \mathrm{O}_{3}$ 表面 酸碱性质与磷酸根吸附研究. 环境科学学报, 2007, 27(4): 637-642.

[9] 孟文娜, 谢杰, 吴德意. 活性氧化铝对水中磷的去除与回收研 究. 环境科学, 2013, 34(1): 231-236.

[10] KIM Y, KIRKPATRICK R J. An investigation of phosphate adsorbed on aluminium oxyhydroxide and oxide phases by nuclear magnetic resonance. European Journal of Soil Science, 2004, 55(2): $243-251$.

[11] JOHNSON B B, IVANOV A V, ANTZUTKIN O N, et al. ${ }^{31} \mathrm{P}$ nuclear magnetic resonance study of the adsorption of phosphate and phenyl phosphates on $\gamma-\mathrm{Al}_{2} \mathrm{O}_{3}$. Langmuir, 2002, 18(4): 1104-1111.

[12] 杨晓芳. 原位红外光谱研究含氧离子氧化物微界面吸附过程与 机理. 北京: 中国科学院大学博士学位论文, 2010 .

[13] 邵兴华, 章永松, 林咸永. 三种铁氧化物的磷吸附解吸特性以 及与磷吸附饱和度的关系. 植物营养与肥料学报, 2006, 12(2): 208-212.

[14] CHATMAN S, ZARZYCKI P, PREOANIN T, et al. Effect of surface site interactions on potentiometric titration of hematite $\left(\alpha-\mathrm{Fe}_{2} \mathrm{O}_{3}\right)$ crystal faces. Journal of Colloid and Interface Science, 2013, 39(1): 125-134.

[15] 严玉鹏. 几种土壤有机磷在铁铝氧化物表面的吸附、解吸与沉 淀. 武汉: 华中农业大学硕士学位论文, 2015.

[16] 杜永, 李斌, 任海萍, 等. 纳米介孔氧化铝制备及表征. 无机盐 工业, 2008, 40(5): 34-35.

[17] DIZ P, MENA A, NOMBELA MIGUEL ÁNGEL, et al. Description of an experimental set up for the culture of benthic foraminifera in controlled $\mathrm{pH}$ conditions. Thalassas: An International Journal of Marine Sciences, 2015, 31(1): 23-32.

[18] 张学清, 项金钟, 胡永茂. 纳米 $\mathrm{Al}_{2} \mathrm{O}_{3}$ 的制备及红外吸收研究. 中国陶瓷, 2004, 40(1): 24-27.

[19] AZIZIAN S. Kinetic models of sorption: a theoretical analysis. Journal of Colloid and Interface Science, 2004, 276(1): 47-52.

[20] 李显波, 马力, 刘志红, 等. 活性氧化铝对废水中磷酸根离子的 吸附特性研究. 非金属矿, 2017, 40(4): 4-7.

[21] 张炳慧. 天然水中磷酸盐存在状态与 $\mathrm{pH}$ 值的关系. 地质实验室, 1992, 8(2): 98-100.

[22] CHUBAR N I, KANIBOLOTSKYY V A, STRELKO V V, et al. Adsorption of phosphate ions on novel inorganic ion exchangers. Colloids and Surfaces A (Physicochemical and Engineering Aspects), 2005, 255(1/2/3): 55-63. 